\title{
Cryogenic Transmission Electron Microscopy Investigation of Carbon Nanothreads
}

Danielle Reifsnyder Hickey ${ }^{1}$, Stephen $\mathrm{Juhl}^{2}$, Arani Biswas ${ }^{3}$, Elizabeth Elacqua ${ }^{3}$, Vincent Crespi ${ }^{3}$, Timothy Strobel ${ }^{4}$ and Nasim Alem ${ }^{3}$

${ }^{1}$ Pennsylvania State University, Pennsylvania, United States, ${ }^{2}$ DuPont, United States, ${ }^{3}$ Pennsylvania State University, United States, ${ }^{4}$ Carnegie Institution for Science, United States

The ability of the element carbon to form many types of chemical bonds enables it to exist as a variety of allotropes, including diamond, graphite/graphene, and fullerenes [1]. Recently, a new class of hydrocarbon materials has been discovered, called carbon nanothreads [2]. Nanothreads have been created by the controlled compression of aromatic molecules, such as benzene and its derivatives, and the resulting structure consists largely of one-dimensional sp3-bonded carbon networks saturated with hydrogen.

X-ray diffraction (XRD) and a variety of chemical characterization techniques have helped to establish the structure and bonding in nanothreads [2], but transmission electron microscopy (TEM) is uniquely able to determine the local, real-space structure [3]. XRD measurements show that benzene-derived nanothreads pack into a pseudohexagonal lattice, with a [10-10] spacing equal to $5.6 \AA$ [2]. However, under the electron beam, damage occurs so quickly that even low-dose TEM conditions ( 5 e/Å2s) at roomtemperature only capture images of an expanded lattice spacing of $5.9 \AA$ after the initiation of beam damage in the lattice [4].

For this reason, analysis at cryogenic temperatures (cryo-TEM) is an attractive avenue to pursue. Here, we show that cryo-TEM has enabled the significant reduction of beam damage in carbon nanothreads. This allows the native structure of benzene-derived carbon nanothreads to be imaged successfully, both in real space and in diffraction mode. This presentation will show successes from using cryo-TEM and a comparison with high-resolution nanothread imaging at room temperature.

Nanothreads can also be synthesized with a variety of compositions, using benzene derivatives as precursors [5-8]. Recently reported precursors have included pyridine [5], thiophene [6], furan [7], and cocrystals $[8,9]$. Beyond what has been achieved for benzene-derived nanothreads, this presentation will further highlight recent results on the structural analysis via TEM of other nanothread chemistries.

The authors gratefully acknowledge funding provided by the Center for Nanothread Chemistry, a National Science Foundation (NSF) Center for Chemical Innovation (CHE-1832471). This work utilized resources provided by the NSF-MRSEC-sponsored Materials Characterization Lab and Huck Institute at Penn State. 
a

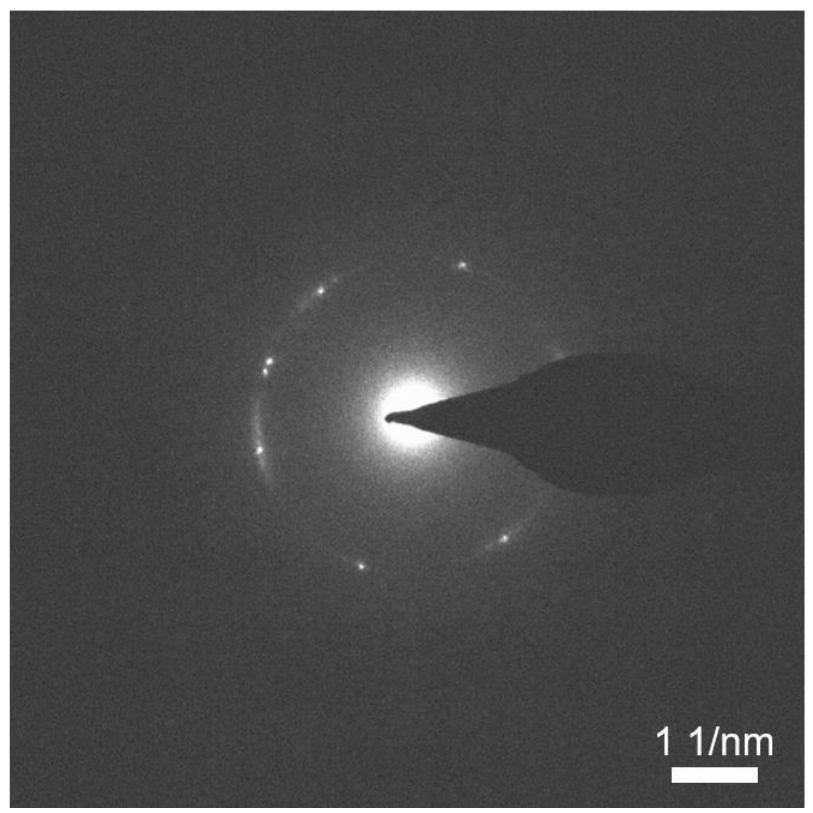

$\mathrm{b}$

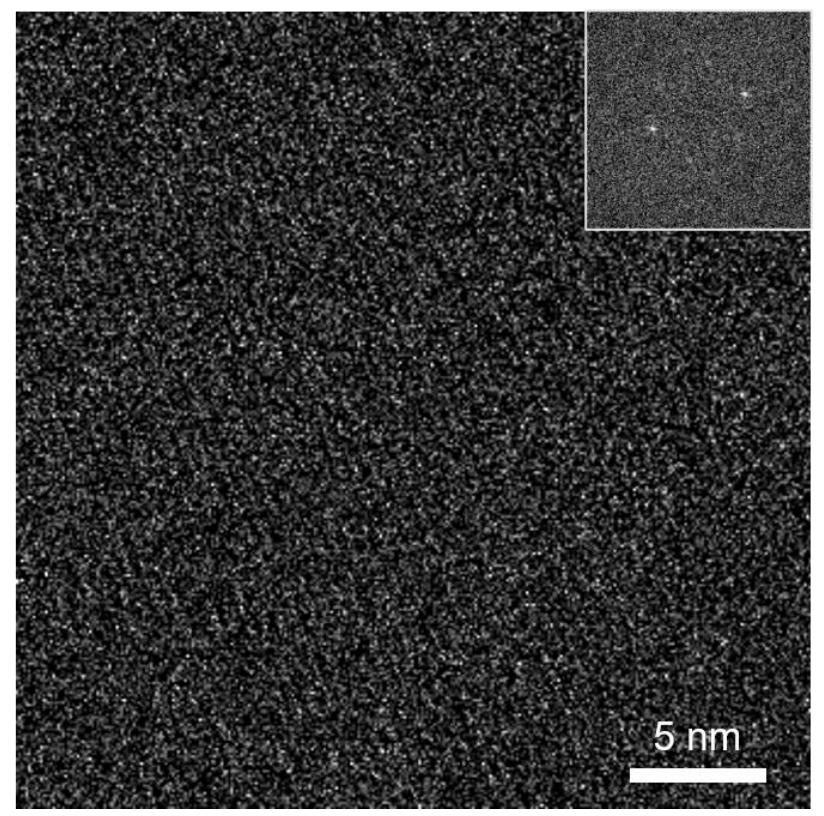

Figure 1. Cryo-TEM characterization of benzene-derived carbon nanothreads. (a) A representative cryoelectron diffraction pattern. (b) Low-dose cryo-high-resolution imaging (inset: FFT).

\section{References}

[1] J. V. Badding et al., SPIE Newsroom (2015). https://doi.org/10.1117/2.1201501.005713.

[2] T. C. Fitzgibbons et al., Nat. Mater. 14 (2015) 43.

[3] S. J. Juhl et al., J. Am. Chem. Soc. 141 (2019) 6937.

[4] S. J. Juhl. Ph.D. Thesis. The Pennsylvania State University (2019).

[5] X. Li et al., J. Am. Chem. Soc. 140 (2018) 4969.

[6] A. Biswas et al. J. Phys. Chem. Lett. 10 (2019) 7164.

[7] S. Huss et al. ACS Nano ASAP.

[8] M. D. Ward et al. Macromolecules 52 (2019) 7557.

[9] M. C. Gerthoffer et al. Chem. Sci. 11 (2020) 11419. 